\title{
DESIGN FOR HEALTH 4.0: EXPLORATION OF A NEW AREA
}

\author{
Bause, Melania; Khayamian Esfahani, Bahar; Forbes, Hannah; Schaefer, Dirk
}

The University of Liverpool

\begin{abstract}
Driven by networked Electronic Health Record systems, Artificial Intelligence, real-time data from wearable devices with an overlay of invisible user interfaces and improved analytics, Health 4.0 is changing the healthcare industry. The focus on collaboration, coherence, and convergence that will make healthcare more predictive and personalised. Furthermore, Health 4.0 realises the value of data more consistently and effectively. It can pinpoint areas of improvement and enable more informed decisions. What it also does is help move the entire healthcare industry from a system that is reactive and focused on fee-for-service to a system that is value-based, which measures outcomes and ensures proactive prevention.

In this paper, the authors will first explore the realm of the emerging area of Health 4.0 and identify its opportunities and challenges. This includes understanding the relevant base technologies as well as the design principles for the realization of smart healthcare product, systems and product-service-systems of the future. Following on from there, the authors focus on the role of design in the specific context of healthcare.
\end{abstract}

Keywords: Health 4.0, Design for health, User centred design, Design for X (DfX), Industry 4.0

\section{Contact:}

Forbes, Hannah

The University of Liverpool

Industrial Design

United Kingdom

hannah.forbes@liverpool.ac.uk

Cite this article: Bause, M., Khayamian Esfahani, B., Forbes, H., Schaefer, D. (2019) 'Design for Health 4.0: 


\section{INTRODUCTION}

A growing ageing population and the rise in the number of people living with long-term conditions is leading to an increasing demand for resources to support healthcare. According to the UK National Statistics (2018), the worldwide population is ageing at a significant rate. In 2015 there were around 901 million people over 60 years of age which is $12.3 \%$ of the world population and further statistics show that by 2030 there will be an increase of 1.4 billion or $16.4 \%$ and by 2050 an increase of 2.1 billion or $21.3 \%$. It is therefore increasingly important to provide an equal and equitable healthcare system for the most vulnerable in our society including the elderly and those in remote areas who have limited access to regular healthcare provision. In the last few years, however, "healthcare organisations have been facing several critical issues, such as patient safety, quality of service, financial constraints and budget reductions." (Merone et al., 2018). Studies have shown that "a significant number of patients are harmed during health care, either resulting in permanent injury, increased length of stay in health care facilities, or even death." (WHO, 2018). Furthermore, as Stuckler et al. (2018) have discovered, a political agenda of austerity, like those many countries in the EU, are adopting, has led to significant job losses in healthcare (Stuckler et al., 2018). This, in turn, has resulted in a "reduced quality of service" and further implications of declining health such as an increase in suicides and homelessness (Stuckler et al., 2018). There is therefore great evidence to suggest that new approaches and solutions to healthcare are required.

The current technological advancements in Information and Communication Technologies (ICT) are now extending to healthcare, which brings about a so-called Health 4.0 revolution. This paradigm shift in healthcare towards technology-enabled care services (TECS) benefits both patients and physicians (NHS, 2018) and its benefit are being recognised with the UK government planning to significantly increase TEC solutions by 2020 (UKGOV, 2018). The plan is to empower patients to access health data and track their information for preventive care, support and treatment (UKGOV, 2014). The empowerment of patients, however, is only one facet of Health 4.0 and the technologies it encompasses in several fields.

This paper focuses on the exploration of the three "pillars" of Health 4.0; people, technology and design. It presents Health 4.0 opportunities and challenges and offers a foundation for the next stage of the research which is concerned with investigating, understanding and assessing the full spectrum of customer requirements from the perspectives of all stakeholders involved. Future research, however, will include further exploration in design research with methods of conducting case studies and interviews.

The remaining part of the paper proceeds as follows: Section 2 is concerned with the review of literature used for this paper, focusing on the identification of the existing definitions of Health 4.0, followed by the role of technology and design in Health 4.0. Section 3 discusses the opportunities and challenges focusing on the research questions. Lastly, section 4 draws together the key findings and opens suggestions for future research.

\section{LITERATURE REVIEW}

As part of the literature review, an overview of the literature field and the areas of research that contribute to the academic outlook on Health 4.0 are described.

\subsection{Defining health $\mathbf{4 . 0}$}

Health 4.0 is defined as "a strategic concept for the health domain derived from the Industry 4.0 concept." The term is often used synonymously with digital health, m-health, e-health and smart health (Thuemmler and Bai, 2017). Behind this concept exists the aim of virtualisation in healthcare and personalization for patients, professionals and other stakeholders and an overall improvement of the health industry with technology. Briefly, Health 4.0 can be described as a phenomenon to improve healthcare service and improve connectivity between health care stakeholders using technology.

As the principal stakeholder of the digitally connected healthcare system, the development of Health 4.0 technology is, firstly, central to meeting patient needs and improving the service patients receive (Syed et al., 2006). To fulfil this, the patient must receive the best and timely medical care possible whenever they need it (Exceet, 2015). This demand has led to the fight for personalisation of health, 
with completely tailored services offering the most benefit to patients. According to Topol's (2015) vision of the future, Health 4.0 will allow patients or "consumers" to be the sole managers of our health due to the "practically endless" data about our bodies, behaviours, and lifestyles that will be collected and analysed (Topol, 2015).

As well as a movement central to patient care, Health 4.0 is a movement designed to increase interconnectivity. Exceet Secure Solutions (2015) indicate the importance of interconnectivity between the main characters in emerging healthcare of the future and according to Tsekleeves and Cooper (2017), an emerging trend is design interaction. They state that in order to improve healthcare, the industry must improve the relationship between people, products, places and services" (Tsekleeves and Cooper, 2017). The implementation of technological trends, therefore, such as the internet of things" will drastically improve worldwide healthcare. In their recent publication on Health 4.0, Mueschenich and Wamperecht (2018) state that digital health in healthcare services are progressing rapidly to the extent that, for the uninformed audience, it remains unseen that processes in care are digitalised daily. Digital medicine is the way forward and by emphasising the drivers such as communication and interaction Health 4.0 can recuperate the imbalance between physician-patient communication (Mueschenich, Wamperecht, 2018).

In the remainder of this literature review, these two aspects of Health 4.0; improved service and improved interconnectivity will be explored with respect to the three "pillars" of Health 4.0; people, technology and design.

\subsection{Health 4.0: People}

For the purpose of this section, the role of patients and physicians, as well as the role of other main stakeholders, is described as part of the smart hospital in health 4.0. Therefore, a vision of a typical Health 4.0 environment, where people give and receive care, is presented.

With regards to a changing hospital environment, Merone et al. (2018) address the importance of redesigning hospital processes and addressing the culture of its staff. They offer a new structure to the traditional model. By analogy with Industry 4.0, which covers the empowerment of change from manufacturer to service provider in order to increase personalisation for clients. Hospitals, as part of Health 4.0, adopt this strategy as "patients and healthcare professionals are strictly connected with the organisation, methodology and the technology" (Merone, 2018). To elaborate, "the major stakeholders in the healthcare system are patients, physicians, employers, insurance companies, pharmaceutical firms and government." (Angell, 2011). All stakeholders of the system function in order to deliver care for the end-users. How these stakeholders are arranged in Health 4.0 differs from the traditional model, shown in Figure 1.

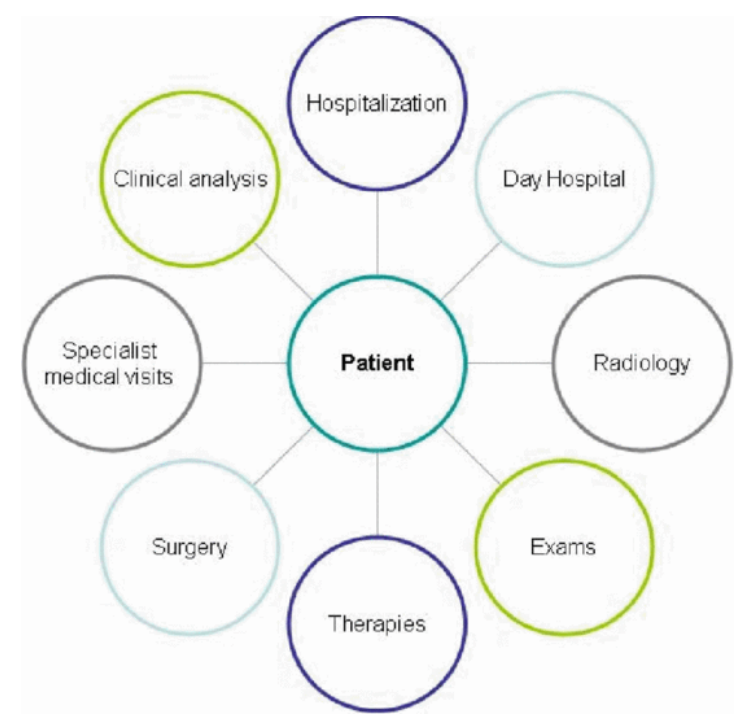

Figure 1. Schematic representation of the poly-functional model typical of a polyclinic. (Merone et al., 2018)

The current approach is a traditional model of a polyclinic where the patient is being taken care of but not seen as the "client of service" (Figure 1). However, the patient experiences limited care and is solely looked after in one specific area, whether that be the GP surgery, at home or at the hospital. In 
the context of Health 4.0, the recipient is the client of service, resulting in an overall better service. In the traditional model, responsibility is with the patient to maintain their medical data and keep track of examinations. This is a burden and often leads to the loss of information, especially in the common case when the patient is of poor health. According to Merone et al. (2018), "the information as provided by the patient may be incomplete, there is also the risk of uncertainty in diagnosis." (Merone et al., 2018).

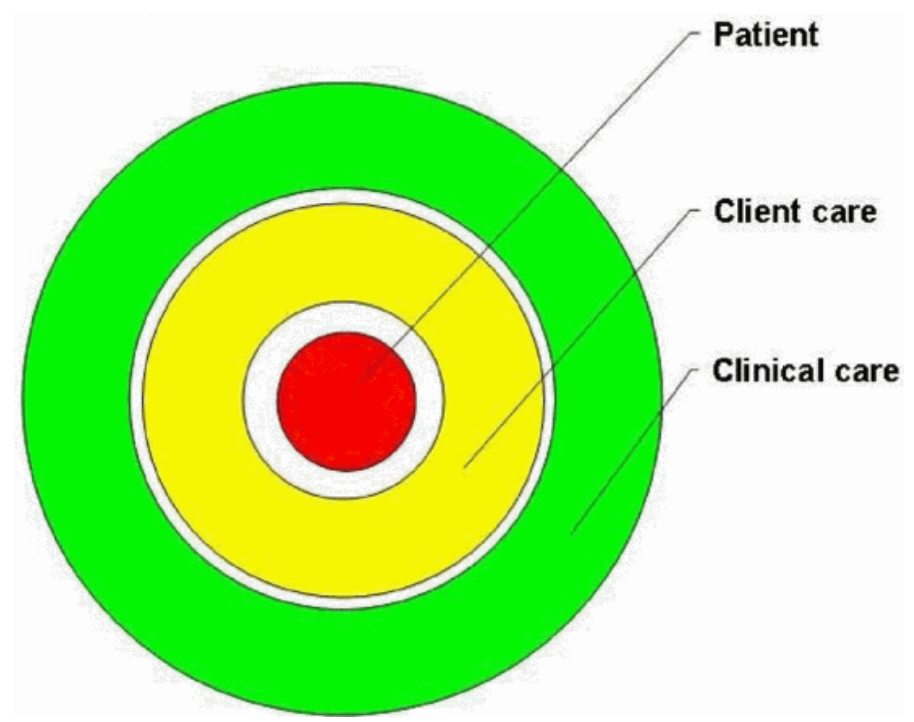

Figure 2. Patient-centred hospital scheme (Merone et al., 2018)

The vision of a 'patient-centred integrated service' in Health 4.0 is where the patient receives effective care without discomfort during investigation and/or therapy. Figure 2 represents this Health 4.0 vision, illustrating the patient as an integrated user. This is designed to avoid needless operations, simplify administrative procedures and, overall, reduce the cost for all stakeholders, particularly the patient. The model suggests the patient to have a single "client care contact point" which usually includes 'a team of several members: physician, nurse, pharmacist, physical therapist and care manager' who oversee procedures concerning the users time spent in hospital. Health 4.0, therefore, represents a new model for stakeholder arrangement in healthcare, where service is conducted in a way that is central to patient needs. This "patient-centralised" service is enabled by technology, the second pillar of Health 4.0, which is described in the next section.

\subsection{Health 4.0: Technology}

To move on from the preceding section, this next part will take a closer look into technology in Health 4.0. According to Thuemmler and Bai (2017) the combined work of cyber-physical systems, IoT (Internet of Things) and smart devices/accessories such as smartphones allows a link between the physical world and the virtual world "to form an Internet of Things, Data and Services." (Thuemmler and Bai, 2017). In healthcare, this link is more specifically described as the link "between wearables, sensors and smart pharmaceuticals to manage diseases" (Thuemmler and Bai, 2017). The results of this link are, briefly, the delivery of personalised healthcare at a reduced cost, as part of Health 4.0. Although, virtualisation means transparency and gathering personal information onto servers that cannot promise immunity against cyber-attacks, which is a major obstacle for deploying credible systems or devices. Nevertheless, Emerging technologies in this new healthcare era, therefore, promise new applications and an enhancement of current applications for examination, diagnosis, therapy and surgery.

Digital technologies can offer limitless possibilities to improve health, from personal fitness to building stronger health systems for entire countries (WHO, 2018). To give an example, The World Health Organisation recently launched the global action plan on physical activity in 2018 and partnered with Google to promote the Google Fit app that shares health advice through new innovative platforms (WHO, 2018) Another example is the partnership between ITU (International Telecommunication Union) and WHO that promotes an m-health solution to combat NCDs (NonCommunicable Diseases) (WHO, 2018). This programme is called "Be He@lthy, Be Mobile" and it has been initiated to connect with the $95 \%$ of the world's online population (WHO, 2018). M-health 
digital platforms are developed for patients and healthcare providers as well as businesses connected to the healthcare system. "They provide a powerful opportunity to extend and change how we access health services, as they can bring them to people in remote areas and can make universal access to healthcare for all a reality-across the globe" (ITU, 2018). Digital technologies on the mobile platform (known as m-health) is therefore already providing patients with enhanced care due to increased connectivity to care providers across the world.

Further research shows that the medical internet of things (IoMT) also plays a major role in providing personalised healthcare and preventative medicine, as stated by Marr (2018) "there are 3.7 million medical devices being used that are connected and monitor body functions and then send evaluations to healthcare professionals" (Marr, 2018). Devices in IoT or the internet of medical things (IoMT) are predicted to grow dramatically in the upcoming years, due to the accessibility of wearables and the decreasing costs of sensor technology (Marr, 2018). As Topol (2015) states "Medicine will be revolutionised by the "Internet of Things", a world of interconnected, sensor-laden devices and objects...sensors have the power to measure our every action...we can profoundly change our behaviour once we are provided with the relevant data" (Topol, 2015).

As well as the application of smart devices in healthcare, further technological advancements are entering the medical sector and improving the way healthcare is and will be delivered. Mesko (2014) focuses on analysing disruptive technologies in medicine and healthcare. He promises technology that will alter how we manage our health and digital technologies that "offer opportunities for doctors and patients to become better strategic partners in medical decisions" (Mesko, 2014). Some technologies that already exist include digestible sensors, micro video cameras for internal diagnosis and smartwatches with the ability to measure and send information to hospital laboratories (Mesko, 2014). Future technology, data and diagnostic tools include embedded sensors such as tooth-embedded sensors that can recognize jaw movement, coughing and speaking (Mesko, 2014). Moreover, augmented reality, in surgery will soon be part of normal practice with Google Glass or digital contact lenses that will be controlled with brain waves (Mesko, 2014). These are some of many examples of cyber-physical systems being applied to the medical sector.

Another advancement, central to the development of other technologies is big data analytics in healthcare. With big data analytics, there is the opportunity to discover association, patterns and future trends to improve healthcare and save lives. All healthcare data is exponentially growing and several "data analysis techniques such as statistical modelling, predictive analytics, artificial intelligence, data mining and machine learning techniques are used in exploration to retrieve effective and efficient patterns from structured and unstructured big data" (Chauhan, Jangade, 2016). Furthermore, as the use of other technologies such as embedded sensors grows, the availability of data will increase as will the opportunity to put data findings into action.

To summarise, Health 4.0 technologies exist as part of $\mathrm{m}$-health, IoT and big data. m-health offers predominantly interconnectivity between patients and healthcare professionals while IoT devices offer the ability to collect information and perform procedures with increasingly minimal invasion. Finally, big data gives healthcare professionals an opportunity to spot trends and patterns for both individual patients and groups of patients, improving the speed of diagnosis and disease prevention. In the next section the third and final pillar of Health 4.0; design, is discussed.

\subsection{Health 4.0: Design}

Design plays a key role in the transformation of smart healthcare systems and how healthcare is delivered with increasing effectiveness and efficiency (Cooper \& Tsekleves, 2017). An integral role of design, in improving smart healthcare systems, starts with understanding and influencing the experience of patients for the best possible outcome. One of the identified current challenges in smart healthcare solutions is that they are often technology-driven without the involvement of the end user (Karen Taylor, 2015). A further challenge remains, therefore, in the enhancement of the patients' participation and engagement in their own health. The incorporation of human-centred design has the potential to reduce risk and ensure patient safety is of the greatest importance. Human-centred design (HCD) is a creative approach to problem-solving that prioritises understanding human needs in order to develop products, services, and systems that are understandable, usable and desirable for people (Norman, 2013). As previously discussed, the rise in the number of people living with long-term conditions and the cost-effectiveness of technology-enabled care services (TECS) has become one of 
the major market growths towards accessible health technologies for patients, carers and health care professionals (NHS, 2018).

This has led to a significant shift in the role of current global design and technology agencies such as Apple, Google and Philips towards digital innovation for the development of innovative and humancentered healthcare with the support of healthcare providers and patients (Apple, 2018; Google, 2018; Philips, 2018). Their partnership with hospitals and pharmaceutical companies has shifted the market growth in the developments of personal health-monitoring systems and personal health platforms. Their current technological innovation provides opportunities in the development of personal health monitoring devices (Jeong et al., 2016).

The shift in personal healthcare systems has opened new avenues for a design that empowers the patients and their self-management through personal devices (GOVUK, 2018). The triangulation between design agencies, technology providers and the stakeholders in the healthcare system, products and systems drives the current healthcare transformation globally. An example of the resulting technologies includes by Google, Apple and Philips for personal health platforms(Karen Taylor, 2015). The health kit enables users to monitor and share their health information such as health conditions, medication, allergies and more. In a similar case, Google has developed personal healthmonitoring platforms for health and activity tracking. In addition, Philips has focused on technological healthcare innovations that meet the end user needs mainly on improving the patients' care.

This recent emerging focus on personalised healthcare has optimised the value of human-centred design to enhance the development of healthcare centred on the patients' experience. This has also raised the contribution of the current global design firms such as IDEO to create innovatively personalised and patient-centered healthcare services (IDEO, 2018). IDEO design strategy incorporates the application of design thinking methodology and human-centred design techniques. Design thinking is a problem-solving approach that enables a better understanding of user needs with innovative problem-solving strategies (Design Council, 2005). In this context, design thinking facilitates the intersection of understanding patients through human-centered design techniques to enhance patients' engagement. Another important aspect of understanding the user/patient experience is related to patient safety rule under the Food and Drug Administration obligation (CDRH, 2017). There is increasing concern over the potential risks for the patients along with the technological advancement in healthcare systems. In order to minimise the potential risks, the integration of human factors principles is required to reduce risk and improve patient safety (Mhra, 2017). The human factor is a scientific discipline that focuses on optimizing user interaction with systems, tools and machines which underpins patient safety in healthcare (NHS, 2013). The incorporation of human factors and design thinking opens up opportunities to create a full spectrum of user requirements for smart healthcare systems designed and delivered safely and effectively.

\section{OPPORTUNITIES AND CHALLENGES}

The digitisation of Health 4.0 is the way forward in healthcare and promises care to be more precise and personalised. Health 4.0 will restructure the healthcare system to the convenience of all stakeholders, most importantly the patient, and it will reduce cost in healthcare. Overall, Health 4.0 looks to be revolutionising healthcare with the latest technology and for the better.

Nevertheless, the interrelation between stakeholders in healthcare is complex and, as discussed by Merone et al. (2018), centring the patient in this system is likely to be initially "chaotic" (Merone et al., 2018) In addition to this culture shift, all stakeholders will have to adjust to new environments, technologies and regulations, which will further complicate this process. The proposed introduction of this technology will see a decrease in productivity which could, as a consequence, lead to a reduced quality of service. The question is therefore whether current stakeholders have the capacity, in an already high-pressure environment, to adapt quickly enough to limit the damage of this monumental change to the healthcare system.

As well as new technologies, healthcare professionals will be expected to conduct their roles with different priorities in mind. For example, as big data becomes more integral to how decisions are made, the patient's own thoughts and feeling on their symptoms could be questioned more easily. In the case when a patients' own description of their symptoms conflicts with their technological description, healthcare professionals have a new challenge in understanding how to resolve this conflict and proceed. 
On this note, "patient sovereignty" will now become an important debate. In particular, the ownership of data in healthcare, while already an important topic of discussion, will become an even more complex argument. As access to data increases, the personalisation and tailoring of healthcare will increase thus improving patient care. The storage and security of personal data as we experience it in social media today will be (and is) as challenging in healthcare, possibly to a greater extent as health information is highly personal matter (Exceet, 2015). All things considered, it is hereby reasonable to say that technology in healthcare is applied to enhance diagnosis, therapy and the overall experience of medical service for patients. However, this change does not implement itself without solving problems "all this is possible only where the patient has given his or her clear, unequivocal consent. The patients retain sovereignty over their medical data at all times" (Exceet, 2015).

When it comes to saving lives, we have briefly spoken about patients' safety and quality of care which leaves room for improvement in design and for healthcare organisations, systems and devices tailored for individuals can enhance health care quality. This vision, however, as demonstrated, is accompanied by a plethora of challenges associated with the right to own personal data, conflict resolution between data and self-diagnosis and significant culture changes in the healthcare system. As Health 4.0 systems are developed, the solutions to these challenges must also be developed with the same fervidity as technological advancements. Without addressing these challenges, the exciting opportunities that Health 4.0 present, cannot become a reality.

Today patients increasingly benefit from digital technology in healthcare and it is increasingly supporting them to better manage and understand their condition. "Online health communities enable patients to share their experiences, swap management tips and exchange resources; apps support medication adherence, symptom tracking and peer support; and there are a host of websites providing health information." (NHS, 2018). With regards to patient processes, design research is applied in; examination, diagnose, treatment and recovery. Patient already benefit from accessing their information on smartphones, tablets and other similar devices that provide access to a number of apps to monitor their health.

In section 2.3 Health 4.0: Technology we have introduced the main emerging technologies and have discovered that these changes will contribute to the way design is delivered in healthcare. A central question is; how does the healthcare sector benefit from the impact of technological advances? The potential impact will allow interconnectivity between healthcare stakeholders, technicians and designers. All in conjunction to provide healthcare services, systems and devices in order to carry out preventative health and treat acute and chronic conditions. But the rigidity of the healthcare structure has the potential to hinder innovation due to its solid standing integral systems. In 2006 the Harvard Business Review published an article by R. E. Herzlinger (2006) on "Why Innovation in Health Care Is So Hard "and state: "Innovations need to appeal to doctors, who are in a position to recommend new products to patients, and doctors' opinions differ.". This is where the focus is to be held, on physician- patient encounter as these are the provider and recipient of future medical care. Further, the article discusses the key forces that affect innovation in healthcare which are "industry players, funding, public policy, technology, customers, and accountability". This shows the dependence of each force to another and implies that various strings play a role in opening up for technology to improve health for patients and environment for the system. Now, to overcome the rigidity of the healthcare structure and with regards to accountability it is suggested to focus on "process rather than on output", looking at improvement in patient health rather than on "whether a provider has followed a treatment process" (hbr, 2006).

Important to include are the healthcare segments in the healthcare industry sector which are divided in four segments (technofunc, 2013):

- Health care services and facilities

- Medical devices, equipment, and hospital supplies manufacturers

- Medical insurance, medical services and managed care

- $\quad$ Pharmaceuticals \& Related Segments

The Healthcare services and facilities segment already shows significant change when it comes to employment in the field, e.g. new career choices such as EEG technologists, MRI technologists or surgical technologists are not unusual today. Design research is involved in all four stages and change of technology requires rethinking design methods especially when designing patient centred systems or devices that require personal data security. From a designers' perspective designing for people is what its profession entails therefore it challenge is not to focus on user needs rather than on 
interconnectivity between services and people needs. Nonetheless, the specialisation of a designer shifts and expertise ads up to the profession, one is required to be of knowledge in business strategies, technology and design.

The authors of this paper have come to its final and pivotal question on; how will the changed technological possibilities impact design research? To be able to answer this question we have to define "what is research good for, and how can we use it for the purpose of design?" (Freach, 2011). Designing nowadays without research is unthinkable, designers thrive from the knowledge they gain and the working concept of how people function. Research in design is the essence to a successful product, as well as it is in satisfying peoples' desirability. In designing for healthcare, the greater challenge would be the gathering of qualitative and quantitative data, but with new technology there is a path for collaboration. Design research is operating on a gigantic platform of information, gathered through the internet, remote video chat studies, connection base around the globe. At the same time these options make the world smaller and a more accessible place. However, the practice of learning about people's behaviour and cultural understanding will not diminish -through new technology- from the traditional way design research is conducted. What mostly has been noticed from the information for this paper is a unified notion amongst authors with regards to technology assisting human lives, a form of coexistence.

\section{CONCLUSION}

Health 4.0 is defined as "a strategic concept for the health domain derived from the Industry 4.0 concept." It is understood to encompass the changes to the way health is managed and delivered due to recent technological advancements. The authors conducted an in-depth analysis and synthesis of Health 4.0 literature and present their findings according to, what they described as, the "three pillars of Health 4.0"; people, technology and design. With regards to people, Health 4.0 is perhaps offering a disruptive solution to the current system. Health 4.0 looks to re-align healthcare stakeholders to make the patient more central to the system. Regarding technology, three areas are most relevant to Health 4.0; m-health, the internet of medical things (IoMT) and big data. m-health offers greater interconnectivity and a stronger relationship between patients and healthcare professionals and patients and their own understanding of their health. IoMT broadly defines the new ways information on health can be collected and healthcare procedures can be conducted. Devices such as embedded sensors and phenomena such as augmented reality are becoming significantly more integrated into healthcare services. Big data is also fundamental to these technological changes in healthcare with faster and more accurate diagnoses being enabled by greater access and availability of data. The third "pillar of Health 4.0", design, refers to the ways in which these new systems are being designed. These design approaches are also new to healthcare, with cutting-edge solutions requiring cutting-edge design approaches. Approaches such as user-centred design and the use of design thinking and human factor principles are now vital to the development of Health 4.0 technologies.

With the growing demand for Health 4.0 technologies comes new challenges to overcome. Health 4.0 demands a "re-centralisation" of the patient in the healthcare system which requires a significant cultural change in the current system. Furthermore, healthcare professionals will be expected to adopt and learn new technologies in an already high-pressured environment which may lead to productivity losses. Finally, "patient sovereignty" with regards to their own personal data is a debate that will continue to grow as Health 4.0 technologies are introduced. As these technologies are improved and implemented, solutions to these challenges must too be addressed for the opportunities of Health 4.0 to be fully capitalised on.

\section{REFERENCES}

A. Storey (2018), "Living longer - how our population is changing and why it matters". [online] Available at: <https://www.ons.gov.uk/peoplepopulationandcommunity/birthsdeathsandmarriages/ageing/articles/livingl ongerhowourpopulationischangingandwhyitmatters/2018-08-13> [Accessed 30.November]

Anon (2013), "Human factors in healthcare a concordat from the national quality board", Available at https://www.england.nhs.uk/wp-content/uploads/2013/11/nqb-hum-fact-concord.pdf [Accessed November 26, 2018].

Apple (2018), "Healthcare - apple (UK)”. Available at: https://www.apple.com/uk/healthcare/ [Accessed November 26, 2018]. 
B. Marr (2018), "Why the internet of medical things will start to transform healthcare in 2018". [online] Forbes. Available at: <https://www.forbes.com/sites/bernardmarr/2018/01/25/why-the-internet-of-medical-thingsiomt-will-start-to-transform-healthcare-in-2018/\#44fabaed4a3c> [Accessed 11. November 2018]

B. Mesko (2014), "Rx disruption: technology trends in medicine and health care". [online] Available at: $<$ https://liverpool.idm.oclc.org/login?url=https://search.ebscohost.com/login.aspx?direct=true \&db=edswss $\& \mathrm{AN}=000334257300011 \&$ site $=$ eds-live $\&$ scope $=$ site $>$ [Accessed 10 November 2018]

BBC, "Diabetes glucose monitors "available to thousands more" - BBC news". Available at: https://www.bbc.co.uk/news/health-46198366 [Accessed November 21, 2018].

C. Thuemmler and C. Bai (2017), Health 4.0: How Virtualization and Big Data are Revolutionizing Healthcare; p. 17, p. 24. Springer International Publishing, Switzerland

CDRH, "Medical device safety action plan: protecting patients, promoting public health", Available at: https:/www.fda.gov/downloads/AboutFDA/CentersOffices/OfficeofMedicalProductsandTobacco/CDRH/ CDRHReports/UCM604690.pdf [Accessed November 26, 2018].

Cooper, E. and Tsekleves, E., "Design for health", Available at: http://radar.gsa.ac.uk/5329/1/9781472457424_chapter 17 Macdonald.pdf [Accessed November 26, 2018].

Design Council (2005), “A study of the design process”. Design Council, Vol. 44 No. 0, pp. 1-144.

E. Tsekleves and R. Cooper (2017), Emerging Trends and the Way Forward in Design in Healthcare: An Expert's Perspective. The Design Journal, London

Exceet AG (2015), "Health 4.0 the digitally connected healthcare system of the future: practical uses: [pdf] exceet”. Available at: <https://www.exceet.com/fileadmin/exceet/downloads/Newsletter/eHealth_Telematics_infrastructure_ENG.pdf> [Accessed 31. October 2018]

Frog Design (2018), "Healthcare service strategy". [online] Available at: <https://www.frogdesign.com/sector/healthcare-service-strategy> [Accessed 1 November 2018]

G. Eysenbach (2001), "What is e-health?” [pdf] JMIR. Available at: <https://asset.jmir.pub/assets/dc6e174866b362a0d994c7c1db6f2dc5.pdf> [Accessed 1 November 2018].

Google, "Google Fit | Google Developers”. Available at: https://developers.google.com/fit/ [Accessed November 26, 2018].

GOVUK, "Personalised health and care 2020: a framework for action - GOV.UK". Available at: https://www.gov.uk/government/publications/personalised-health-and-care-2020/using-data-andtechnology-to-transform-outcomes-for-patients-and-citizens [Accessed November 20, 2018].

IDEO (2018), "How will we personalize healthcare?" | ideo.com. Available at: https://www.ideo.com/question/how-might-we-transform-institutionalized-care-into-individualized-care [Accessed November 26, 2018].

International Telecommunication Union (2018), "M-health for NCD". [online] Available at: <https://www.itu.int/en/ITU-D/ICT-Applications/Pages/mhealth-for-ncd-behealthy-bemobile.aspx> [Accessed 14. November 2018]

Jeong, J.-S., Han, O. and You, Y.-Y. (2016), “A design characteristics of smart healthcare system as the IoT application”. Indian Journal of Science and Technology, Vol. 9 No. 37. Available at: http://www.indjst.org/index.php/indjst/article/view/102547 [Accessed November 26, 2018].

J. Freach (2011), “The art of design research and why it matters". [online] Available at: $<$ https://www.theatlantic.com/entertainment/archive/2011/05/the-art-of-design-research-and-why-itmatters/239561/> [Accessed 18 March 2019]

K. Taylor (2017), [online] Available at: <https://www.futurehealthindex.com/2017/06/13/by-2020-the-smarthospital-will-be-a-reality/> [Accessed 19 October 2018]

Karen Taylor (2015), “The deloitte centre for health solutions”, Available at: https://www2.deloitte.com/content/dam/Deloitte/uk/Documents/life-sciences-health-care/deloitte-ukconnected-health.pdf [Accessed November 20, 2018].

M. Angell (2011), "Health care reform: duties and responsibilities of the stakeholders". [online] SJU. Available at: <https://sites.sju.edu/icb/health-care-reform-duties-and-responsibilities-of-the-stakeholders/> [Accessed 10 November 2018]

M. Merone, P. Afferni and P. Soda (2018), "Hospital 4.0 and its innovation in methodologies and technologies". [online] IEEE. Available at: https://ieeexplore-ieee-org.liverpool.idm.oclc.org/document/8417260 [Accessed 2. November 2018]

M. Mueschenich and L. Wamprecht (2017), Health 4.0 - How are We Doing Tomorrow? Springer Verlag GmbH, Germany

M. Pferzinger and P. Rammerstorfer (2017), Design Thinking Based Digital Transformation in Healthcare. Springer Fachmedien Wiesbaden GmbH, Germany

Mhra (2017), "Human factors and usability engineering-guidance for medical devices including drug-device combination products human factors and usability engineering-guidance for medical devices including drug-device combination products MHRA", Available at: http://www.nationalarchives.gov.uk/doc/opengovernment-licence/ [Accessed November 26, 2018]. 
NHS, "NHS England» Technology Enabled Care Services (TECS)”. (2018), Available at: https://www.england.nhs.uk/tecs/ [Accessed November 26, 2018].

PHILIPS, "Innovating meaningful healthcare $\mid$ philips healthcare". Available at: https://www.philips.co.uk/healthcare [Accessed November 26, 2018].

R. E. Herzlinger (2006), "Why innovation in healthcare is so hard". [online]. Available at: <https://hbr.org/2006/05/why-innovation-in-health-care-is-so-hard> [Accessed 19 March 2019]

R. Chauhan and R. Jangade (2016), “A robust model for big healthcare data analytics". [pdf] Available at: <https://ieeexplore-ieee- org.liverpool.idm.oclc.org/stamp/stamp.jsp?tp=\&arnumber=7508117\&tag=1> [Accessed 10 November 2018]

Reid, R.J., Coleman, K., Johnson, E.A., Fishman, P.A., Hsu, C., Soman, M.P., Trescott, C.E., Erikson, M. and Larson, E.B., 2010. "The group health medical home at year two: cost savings, higher patient satisfaction, and less burnout for providers". [pdf] Available at: <https://pdfs.semanticscholar.org/c76d/aef075446cd0ccb5bc13ba50e84d2ff672e8.pdf> [Accessed 20 March 2019]

Sophie Castle-Clarke (2018), "What will technology mean for the NHS". [online]. Available at: <https://www.health.org.uk/sites/default/files/NHS-70-What-will-new-technology-mean-for-theNHS.pdf> [Accessed 18 March 2019]

Stuckler, D., Reeves, A., Loopstra, R., Karanikolos, M. and McKee, M. (2018), "Austerity and health: the impact of crisis in the UK and the rest of Europe". Europe's Crises, p. 127.

Technofunc (2013), "Sectors of healthcare industry". [online] technofunc. Available at: <http://www.technofunc.com/index.php/domain-knowledge/healthcare-industry/item/sectors-ofhealthcare-industry> [Accessed 5.03.2019]

Topol, E.J. (2015), The Patient will See You Now: The Future of Medicine is in Your Hands, Vol. 2015364. Basic Books, New York.

World Health Organisation (2016), "Global diffusion of eHealth". [pdf] WHO. Available at: <http://apps.who.int/iris/bitstream/handle/10665/252529/9789241511780eng.pdf?sequence=1\#page=16> [Accessed 14. November 2018]

World Health Organisation (2018), "Digital Health, promoting health, far and wide, in the 21st century", [online] Available at: 〈http://www.who.int/behealthy/digital-health/promoting-health-in-the-21st-century> [Accessed 14. November 2018]

World Health Organisation (2018), "Patient safety". [online] WHO. Available at https://www.who.int/patientsafety/en/ [Accessed 1. November 2018]

World Health Organisation (2018), "Seventy-First world health assembly". [pdf] WHO. Available at: <http://apps.who.int/gb/ebwha/pdf_files/WHA71/A71_R7-en.pdf> [Accessed 14 November 2018] 